\title{
Entanglement-Assisted Absorption Spectroscopy
}

\author{
Haowei Shi, ${ }^{1}$ Zheshen Zhang, ${ }^{2,1}$ Stefano Pirandola, ${ }^{3}$ and Quntao Zhuang $\oplus^{4,1, *}$ \\ ${ }^{1}$ James C. Wyant College of Optical Sciences, University of Arizona, Tucson, Arizona 85721, USA \\ ${ }^{2}$ Department of Materials Science and Engineering, University of Arizona, Tucson, Arizona 85721, USA \\ ${ }^{3}$ Department of Computer Science, University of York, York YO10 5GH, United Kingdom \\ ${ }^{4}$ Department of Electrical and Computer Engineering, University of Arizona, Tucson, Arizona 85721, USA
}

(Received 28 April 2020; accepted 24 September 2020; published 28 October 2020)

\begin{abstract}
Spectroscopy is an important tool for probing the properties of materials, chemicals, and biological samples. We design a practical transmitter-receiver system that exploits entanglement to achieve a provable quantum advantage over all spectroscopic schemes based on classical sources. To probe the absorption spectra, modeled as a pattern of transmissivities among different frequency modes, we employ broadband signal-idler pairs in two-mode squeezed vacuum states. At the receiver side, we apply photodetection after optical parametric amplification. Finally, we perform a maximum likelihood decision test on the measurement results, achieving an error probability orders of magnitude lower than the optimum classical systems in various examples, including "wine tasting" and "drug testing" where real molecules are considered. In detecting the presence of an absorption line, our quantum scheme achieves the optimum performance allowed by quantum mechanics. The quantum advantage in our system is robust against noise and loss, which makes near-term experimental demonstration possible.
\end{abstract}

DOI: 10.1103/PhysRevLett.125.180502

Quantum sensing and metrology [1-4] harness nonclassical resources to substantially improve the performance of positioning and timing [5], target detection [6-11], digital reading [12], photometry [13,14], distributed sensing [15-20], and most prominently the Laser Interferometer Gravitational Wave Observatory [21-23]. There have been attempts to develop quantum-metrology protocols [24,25] for spectroscopy [26-30], an indispensable tool for science and industry. In this regard, the entangled NOON state [31,32] and its generalizations have been considered in interferometric estimation of loss and phase [33]. However, NOON states are hard to generate and lack robustness against imperfections. As a more experimentally accessible approach, entangled photons produced by spontaneous parametric down conversion have been used for loss estimation [34-39]. In particular, Refs. [37-39] reported nonlinear interferometric probing of midinfrared absorption lines using visible photons. Despite the technical benefits, the quantum advantage over the classical schemes remains unclear.

In this Letter, we investigate entanglement-assisted absorption spectroscopy (EAAS) as an effective means to achieve a provable quantum advantage over all schemes

Published by the American Physical Society under the terms of the Creative Commons Attribution 4.0 International license. Further distribution of this work must maintain attribution to the author(s) and the published article's title, journal citation, and DOI. using classical sources. As depicted in Fig. 1, EAAS uses a source of multichromatic entangled signal-idler mode pairs from a nonlinear media, each being in a two-mode squeezed vacuum (TMSV) state and anticorrelated in the frequency domain. The signals with different frequencies interact with the sample and experience absorption differently, while the idlers are stored locally. Then an optical parametric amplifier (OPA) is applied on the return signalidler pairs, followed by photodetection to classify samples among a plural of possible compositions.

EAAS achieves a strict quantum advantage in the discrimination of arbitrary absorption patterns. Before addressing the general case, we begin with two basic models: absorption detection - the binary testing of a single absorption line at a specific frequency, and peak positioning-pinpointing a given number of absorption lines within a frequency spectrum. Then we consider the classification

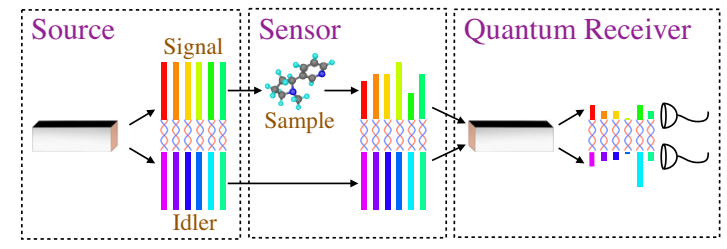

FIG. 1. Diagram of the entanglement-assisted absorption spectroscopy. The source generates multichromatic entangled signalidler pairs via a nonlinear process. The signals interact with the sample and then go through another nonlinear process jointly with the idlers at the quantum receiver. Photodetection extracts the absorption coefficients at different frequencies. 
of several large organic molecules and use real spectrum data [40] to simulate the performance against the optimum classical performance in "wine tasting" and "drug testing." Let us remark that all components in our EAAS are off the shelf, and the quantum advantage is robust against excess noise and idler storage loss, making experimental implementations possible in the near term.

Pattern recognition on absorption spectra.-In absorption spectroscopy [26], each specific composition is associated with a unique absorption spectrum determined by measuring the transmissivities across the spectrum of the input light. Therefore, the overall problem of composition identification can be formulated as a hypothesis testing between several known patterns of the frequencydependent transmissivities, as formulated below.

The multichromatic input light is decomposed into $m$ discrete frequency modes, denoted by the annihilation operators $\left\{a_{\ell}\right\}_{\ell=1}^{m}$. The input-output relation for each mode $a_{\ell}$ is modeled as a thermal loss channel $\mathcal{L}^{\kappa_{\ell}, N_{B}}$ [41] described by the Bogoliubov transformation

$$
a_{\ell} \rightarrow \sqrt{\kappa_{\ell}} a_{\ell}+\sqrt{1-\kappa_{\ell}} e_{\ell},
$$

where $\kappa_{\ell}$ is the transmissivity and $e_{\ell}$ is a thermal mode with mean photon number $N_{B} /\left(1-\kappa_{\ell}\right)$ to model the environmental thermal noise, which is negligible $\left(N_{B} \sim 0\right)$ at the optical wavelengths. However, to demonstrate the robustness of the quantum advantage, $N_{B}>0$ is considered for generality.

The pattern of the transmissivity coefficient $\left\{\kappa_{\ell}\right\}_{\ell=1}^{m}$ reveals the sample's absorption spectrum. We usually have prior information about the possible patterns; therefore, the task is to discriminate between $H$ patterns, each described by a vector $\boldsymbol{\kappa}^{(h)}=\left\{\kappa_{\ell}^{(h)}\right\}_{\ell=1}^{m}$ of transmissivities, where $1 \leq$ $h \leq H$ is the index of the hypotheses and $\ell$ is the frequency-mode index (see Ref. [42] for a channel formulation). In general, we allow $M$ repetitions of the probing attempt to make a decision.

Before addressing the general pattern-recognition problem described above (see Fig. 1), we consider two simplified problems: absorption detection and peak positioning.

In absorption detection, the goal is to determine whether absorption occurs at a single frequency mode $(m=1)$; therefore, there are $H=2$ hypotheses, with transmissivities $\kappa_{B}$ and $\kappa_{T}$ corresponding to the absence and the presence of absorption. In peak positioning, one aims to pinpoint a single absorption peak (target) within $m$ frequencies; therefore, we have $H=m$ possible patterns. Each pattern $h$ has a single absorption peak with transmissivity $\kappa_{T}$ for frequency mode $a_{h}$, while all other frequency modes see a background transmissivity $\kappa_{B}$, i.e., $\kappa_{\ell}^{(h)}=\kappa_{T}$ if $\ell=h$ and $\kappa_{B}$ otherwise.

The problem of absorption detection can be generalized to finding the positions of $k$ absorption peaks in a spectrum of $m$ frequencies, which we call " $k$-peak positioning." In this more general problem, $k$ targets with transmissivity $\kappa_{T}$ are hidden among $m-k$ backgrounds of transmissivity $\kappa_{B}$, so that we have a total of $H=C_{m}^{k}$ hypotheses, where $C_{m}^{k}$ is the binomial coefficient of $m$-choose- $k$. Note that, while we consider these simple examples to introduce our results, our methodology applies to the recognition of arbitrary patterns such as the complex molecules considered at the end of this Letter.

Classical lower bounds.-In a classical spectroscopy scheme, one sends an arbitrary mixture of coherent states as input state. Given $m M N_{S}$ mean total number of photons at the input, where $N_{S}$ is the average mean photon number per frequency mode, the minimum error probability affecting the discrimination between the ensemble of patterns $\left\{\boldsymbol{\kappa}^{(h)}\right\}_{h=1}^{H}$ is lower bounded by

$$
\begin{aligned}
& P_{C, m, L B}=\frac{2}{(H-1) H^{3}} \\
& \quad \times\left(\min _{\left\{X_{\ell}\right\}} \sum_{h^{\prime}>h} \exp \left\{-\frac{1}{2} \sum_{\ell=1}^{m}\left[\sqrt{\kappa_{\ell}^{(h)}}-\sqrt{\kappa_{\ell}^{\left(h^{\prime}\right)}}\right]^{2} \nu_{B} X_{\ell}\right\}\right)^{2},
\end{aligned}
$$

where $\nu_{B}=1 /\left(1+2 N_{B}\right)$ and the minimization is under the energy constraint $\sum_{\ell=1}^{m} X_{\ell} \leq m M N_{S}$ (see [42] for a proof). Applying Eq. (2) to the absorption detection case, we obtain the lower bound $P_{C, 1, L B}=e^{-\nu_{B} M N_{S}\left(\sqrt{\kappa_{B}}-\sqrt{\kappa_{T}}\right)^{2}} / 4$. In this case, a slightly improved bound can be obtained [12]:

$$
P_{C, 1, L B}=\frac{1}{2}\left[1-\sqrt{1-e^{-\nu_{B} M N_{S}\left(\sqrt{\kappa_{B}}-\sqrt{\kappa_{T}}\right)^{2}}}\right] .
$$

Specifying Eq. (2) to the problem of $k$-peak positioning, one obtains [42]

$$
P_{C, m, L B}=\frac{C_{m}^{k}-1}{2 C_{m}^{k}} e^{-2 w_{m, k} \nu_{B} M N_{S}\left(\sqrt{\kappa_{B}}-\sqrt{\kappa_{T}}\right)^{2}},
$$

where $w_{m, k}=k C_{m-1}^{k} /\left(C_{m}^{k}-1\right)$. The latter term is equal to 1 for a single peak, and $w_{m, k} \simeq k(1-k / m)$ for $k$ peaks. When $N_{B}=0$, the lower bound is tight in the error exponent for absorption detection and 1-peak positioning.

Entanglement-assisted strategy.-To achieve a quantum advantage, we exploit entanglement at the input, as given by $M$ copies of a TMSV state $\phi_{M E}$ for each signal-idler pair [42]. Each idler mode is stored locally, with imperfections modeled as a pure-loss channel $\mathcal{L}^{\kappa_{I}, 0}$ of transmissivity $\kappa_{I}$ (with a mode transformation $a_{I} \rightarrow \sqrt{\kappa_{I}} a_{I}+\sqrt{1-\kappa_{I}} v$ and $v$ being a vacuum mode), while the signal modes are sent to probe the patterns. For the special binary case of absorption detection, the error probability is bounded by the asymptotically tight quantum Chernoff bound (QCB), which can 
be efficiently calculated [50,51] from the return Gaussian states $\Xi^{(T / B)}$ composed of $M$ identical copies of $\mathcal{L}^{\kappa_{T / B}, N_{B}} \otimes \mathcal{L}^{\kappa_{I}, 0}\left(\phi_{M E}\right)$. For the general pattern case, a simple tool like the QCB is missing, and, for this reason, we need to design an explicit receiver that is able to show a quantum advantage.

Entanglement-assisted receiver design.-We begin our description of the receiver design with a simple case so as to provide its basic modus operandi. Consider the ideal case of $\kappa_{B}=\kappa_{I}=1$ and $N_{B}=0$. Then the returned state $\Xi^{(B)}=\phi_{M E}^{\otimes M}$ consists of $M$ copies of the ideal TMSV state (while $\Xi^{(T)}$ is mixed because $\kappa_{T}<1$ ). Suppose that we perform a two-mode squeezing (TMS) operation $\mathcal{S}$ (via an OPA), that precisely antisqueezes each TMSV state $\phi_{M E}$. Then we can "null" $\mathcal{S}\left(\Xi^{(B)}\right)$ to tensor products of vacuum, while $\mathcal{S}\left(\Xi^{(T)}\right)$ is nonvacuum. Therefore, a simple photon counting on all the signals and idlers after the TMS operation can identify the input state $\Xi^{(B)}$ if there is any photon count. Errors only occur if we obtain a zero count on $\mathcal{S}\left(\Xi^{(T)}\right)$ : when this happens, we can only guess randomly, with an error probability $R_{m}$. Note that this nulling strategy has been used in classical schemes [42], whose performance is bounded by Eqs. (3) and (4). OPA has also been used in quantum illumination [52], however without exploiting correlations in the patterns.

Let us use a compact notation, where $m=1$ corresponds to absorption detection, for which $R_{1}=1 / 2$, and $m \geq 2$ corresponds to single-peak positioning, with one copy of $\mathcal{S}\left(\Xi^{(T)}\right)$ among $m-1$ copies of $\mathcal{S}\left(\Xi^{(B)}\right)$ so that $R_{m}=(m-1) / m$. Accounting for the zero counts, the error probability for absorption detection $(m=1)$ and single-peak positioning $(m \geq 2)$ is given by [42]

$$
P_{E, m}=R_{m}\left[\frac{1}{1+N_{S}\left(1-\sqrt{\kappa_{T}}\right)}\right]^{2 M} \text {. }
$$

When $N_{S} \ll 1$ and $M \gg 1$, we have $P_{E, m} \simeq$ $R_{m} \exp \left[-2 M N_{S}\left(1-\sqrt{\kappa_{T}}\right)\right]$. Comparing this with the classical lower bounds in Eqs. (3) and (4), we see that EAAS has an exponential advantage: $P_{E, m} / P_{C, m, L B} \simeq$ $2 \exp \left[-M N_{S}\left(1-\kappa_{T}\right)\right]$ for absorption detection, and $\simeq 2 \exp \left[-2 M N_{S}\left(\sqrt{\kappa_{T}}-\kappa_{T}\right)\right]$ for single-peak positioning. In fact, Eq. (5) achieves the QCB [42] and therefore it is optimal for absorption detection.

The above receiver design, and the resulting entanglement advantage, can be generalized to cope with more complex spectrum patterns and the presence of noise and idler loss $\left(N_{B}>0, \kappa_{I}<1\right)$, as described by the following strategy (see Fig. 2): (i) Apply TMS operation with gain $G_{\ell}$ to each of the return signal-idler pairs $a_{S \ell}^{\prime \prime}, a_{I \ell}^{\prime \prime}$ to obtain new modes $a_{S \ell}=\sqrt{G_{\ell}} a_{S \ell}^{\prime \prime}-\sqrt{G_{\ell}-1} a_{S \ell}^{\dagger}$ and $a_{I \ell}=\sqrt{G} a_{I \ell}^{\prime \prime}-\sqrt{G-1} a_{S \ell}^{\dagger}$. (ii) Perform photon counting measurement on all signal and idler modes $\left\{a_{S \ell}, a_{I \ell}\right\}$ to obtain the results as two vectors $\boldsymbol{n}_{S}$ and $\boldsymbol{n}_{I}$. (iii) Finally,

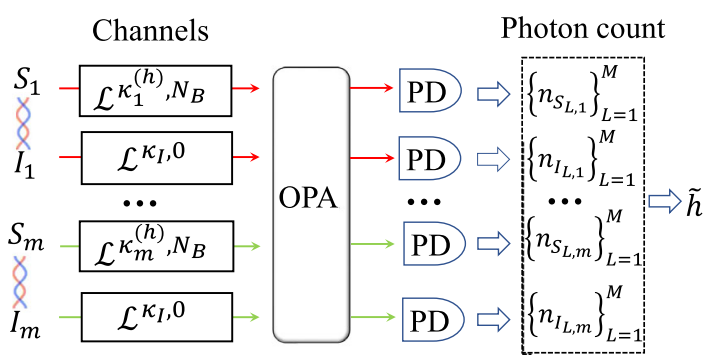

FIG. 2. Schematic of receiver. Signal beams are irradiated over the sample, modeled by frequency-dependent transmissivities $\boldsymbol{\kappa}^{(h)}=\left\{\kappa_{\ell}^{(h)}\right\}_{\ell=1}^{m}$. The modulated beams go through a single optical parametric amplifier. Finally spectrally resolving photodetection offers a $2 m M$-dimensional count based on which the maximum likelihood decision $\tilde{h}$ is made.

apply maximum likelihood decision rule, i.e., make the decision $\tilde{h}$ through

$$
\tilde{h}=\arg \max _{h} P_{m}\left(\boldsymbol{n}_{S}, \boldsymbol{n}_{I} \mid h\right),
$$

where $P_{m}\left(\boldsymbol{n}_{S}, \boldsymbol{n}_{I} \mid h\right)$ is the conditional probability of obtaining the outcomes $\boldsymbol{n}_{S}, \boldsymbol{n}_{I}$ if the true hypothesis is $h$.

To complete the description of our receiver, we need to determine the gain $G_{\ell}$ and specify the conditional probabilities. Let us begin with the cases of absorption detection and peak positioning, where we adopt uniform gain $G_{\ell}=G$. The ideal situation is to get a quantum state close to vacuum; however, if $\kappa_{B}<1$, it is only possible to reduce the signal part of $\Xi^{(B)}$ to vacuum by choosing $G=1+N_{S} \kappa_{B} /\left[1+N_{S}\left(1-\kappa_{B}\right)\right]$. In the presence of noise $N_{B}>0$ and idler loss $1-\kappa_{I}>0$, "nulling" to vacuum is not possible, but the same choice of gain still provides an appreciable advantage over classical schemes. For general patterns, due to the absence of symmetry, we consider optimization over the gain $G_{\ell}$ at different frequency modes. Moreover, as some frequency windows may contain more essential information about the hypotheses, we also allow the optimization over the energy distribution $\left\{N_{S \ell}\right\}$ of the TMSV in different frequency modes. In these cases, although the "nulling" decision rule does not apply, the maximum likelihood decision rule in Eq. (6) still leads to an advantage [42].

Now let us compute the conditional probabilities. With $M$ identical repetitions, the probability of obtaining the $m M$-dimensional measurement results $\boldsymbol{n}_{S}=\left\{n_{S_{L}, k}\right\}_{L=1, k=1}^{M, m}$ and $\boldsymbol{n}_{I}=\left\{n_{I_{L}, k}\right\}_{L=1, k=1}^{M, m}$, conditioned on pattern $h$, is

$P_{m}\left(\boldsymbol{n}_{S}, \boldsymbol{n}_{I} \mid h\right)=\prod_{L=1}^{M} \prod_{\ell=1}^{m} P\left[n_{S_{L, \ell},}, n_{I_{L, \ell}} \mid \kappa_{\ell}^{(h)}, G_{\ell}, N_{S \ell}\right]$,

where each term is a function of the subsystem transmissivity $\kappa_{\ell}^{(h)}$, the TMSV source energy $N_{S \ell}$, and the gain choice $G_{\ell}[42]$. 


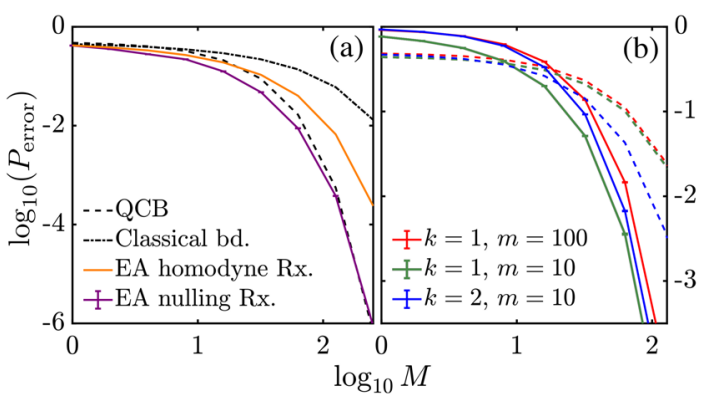

FIG. 3. Error rate versus number of probing modes with practical parameters $N_{S}=1, \kappa_{T}=0.75$, and $\kappa_{B}=0.95$. (a) Absorption detection. EA nulling receiver (solid orange) is compared to classical lower bound of Eq. (3) (dot-dashed black), QCB (dashed black), and the EA homodyne receiver (solid purple). (b) Peak positioning. Single-peak positioning (solid green) and double-peak positioning (solid blue) with $m=10$ frequency slots. Single-peak positioning with $m=100$ (solid red) provided as a reference. Classical lower bounds given by Eq. (4) (dashed, accordingly colored).

With all these theoretical elements in our hands, we can numerically evaluate the error probability $P_{E, m}$ for the problems of absorption detection, peak positioning, and general spectrum recognition via Monte Carlo simulations [42]. Although we consider equal priors for simplicity, our maximum likelihood decision can generally be applied to arbitrary prior probabilities for the patterns.

Detecting and positioning absorption peaks.-In order to investigate the problems of absorption detection and peak positioning, we assume a background transmissivity $\kappa_{B}=0.95$ and a target transmissivity $\kappa_{T}=0.75$. In particular, we study their error probabilities in terms of the number of modes $M$. For absorption detection, Fig. 3(a) shows that our EA nulling receiver asymptotically achieves the QCB [12], outperforming both the best known receiver, the EA homodyne receiver [42,51], and the classical lower bound of Eq. (3). In fact, we can verify that our receiver can asymptotically saturate the QCB for absorption detection with general choices of $\kappa_{B}$ and $\kappa_{T}$ [42]. For peak positioning, as shown in Fig. 3(b), our EA receiver is able to outperform the classical lower bound of Eq. (4) by orders of magnitude.

In a practical scenario, we are interested in how much EAAS can enhance the performance when classical schemes fail to perform well. To showcase the advantage, in Fig. 4 we fix the classical lower bounds in Eqs. (3) and (4) to be 0.01 and plot the error probability $P_{E, m}$ of EAAS. We start with tuning the transmissivities $\kappa_{B}$ and $\kappa_{T}$ in Fig. 4(a),(b). Then we fix $\kappa_{T}=0.75$ and $\kappa_{B}=0.95$ and study how the quantum advantage varies with idler loss $1-\kappa_{I}$ and noise $N_{B}$ in Fig. 4(c),(d). The white dashed lines divide the parameter space with or without quantum advantage. We can see that the advantage is remarkable and also survives for a large range of parameters, especially when $\kappa_{B} \simeq 1$ as in practice. The robustness of the
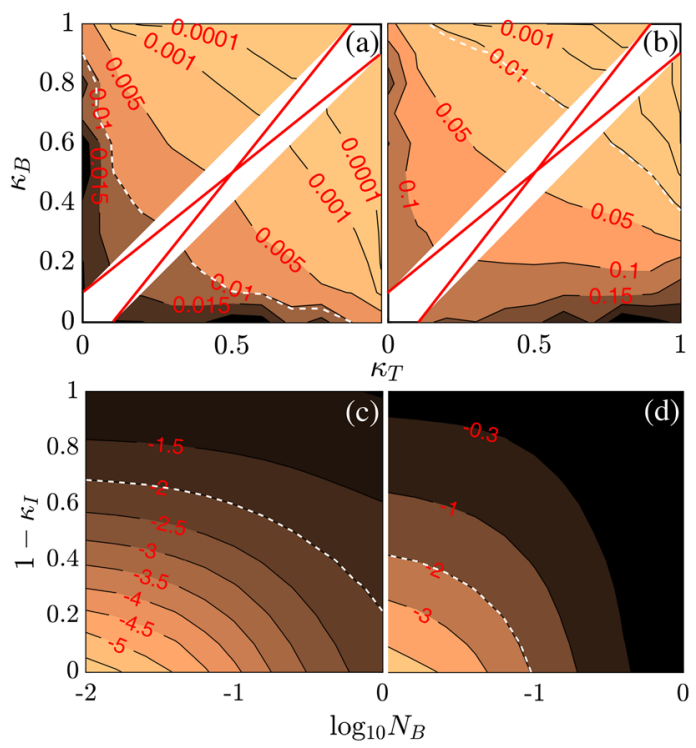

FIG. 4. (a),(b) Error probability of EAAS $P_{E, m}$ versus transmissivities $\kappa_{B}$ and $\kappa_{T}$. (c),(d) $\log _{10} P_{E, m}$ versus idler loss $1-\kappa_{I}$ and thermal noise $N_{B}$ at fixed $\kappa_{T}=0.75$ and $\kappa_{B}=0.95$. Absorption detection $(m=1)$ in (a),(c) compared with singlepeak positioning ( $m=100)$ in (b), (d). $M$ is chosen to fixed the classical lower bounds to 0.01 [42]. $N_{S}=1$ is assumed. Redcrossed diagonal region in (a),(b) represents the degenerate case $\kappa_{B}=\kappa_{T}$.

advantages to imperfections reveals a clear possibility for a near-term experimental demonstration. See Figs. 6-9 in [42] for more parameter settings.

General spectrum recognition.-EAAS can also identify actual molecules, each of which is associated with a unique absorption spectrum. As a taste of flavor, we begin with "wine tasting," where one discriminates three common alcohol-like liquids. Methanol could be lethal if mistaken for ethanol (alcohol). Meanwhile, the alcohol, as time goes by, will be dehydrogenated to ethanal, whose concentration provides the age of a vintage [53]. To consider larger molecules, the second example, "drug testing," involves three drugs: phenyl salicylate, methyl salicylate, and benzoic acid. In both examples, a nondestructive testing method is preferred, therefore we adopt the extremely weak quantum light source. The transmissivities are taken from real Fourier-transform infrared spectra [40]. These spectra are sampled by averaging them within each of $m=4$ frequency slots [42].

As the classical benchmark, we calculate the ultimate lower bound using Eq. (2) and the performance of a homodyne receiver on coherent-state input with the same energy distribution (distribution of mean photon number over frequency modes) optimized in Eq. (2). Figure 5 shows that EAAS with uniform energy distribution, and $G=1$ (orange) outperforms the homodyne receiver (black solid) in both cases. Then, in drug testing, EAAS beats the classical lower bound by orders of magnitude, while in 


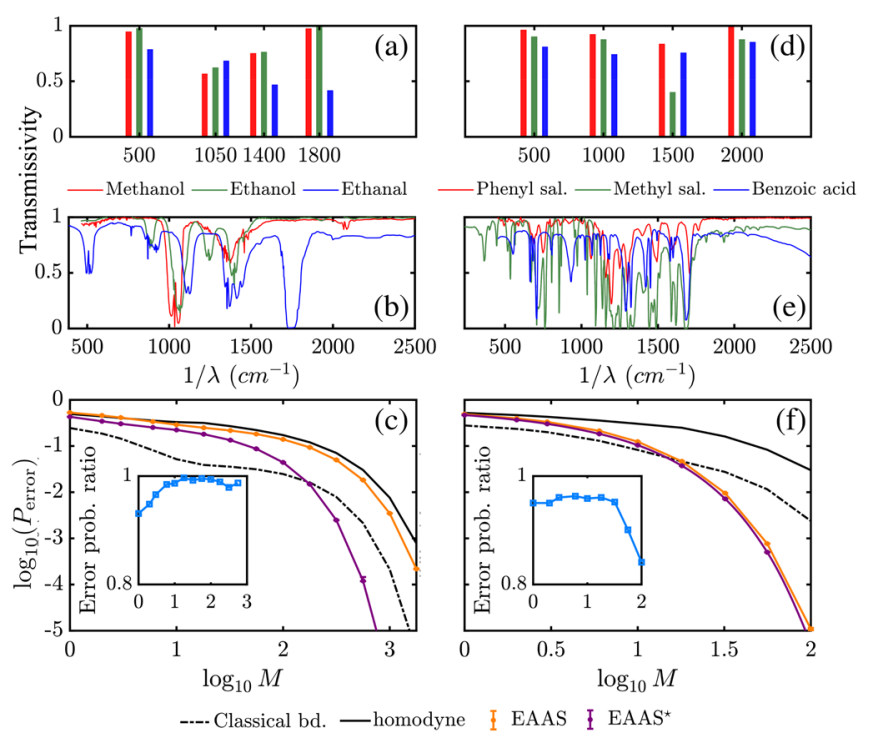

FIG. 5. Identification of $H=3$ molecules with $m=4$ frequency slots for wine tasting in (a),(b),(c) and drug testing in (d),(e),(f). (a),(d) are $m=4$ sampled discrete spectra on the fourier-transform infrared spectra in (b),(e). (c),(f) show the logarithmic error rate of EAAS, with $N_{S}=1$. "EAAS" assumes a uniform distribution of photons at the input modes (solid orange), while numerically optimized energy distribution is presented in "EAAS" (solid purple). The classical lower bound (dot-dashed black) and homodyne detection (solid black). Insets of (c),(f): Error probability ratio of EAAS with gain optimization after energy-distribution optimization and EAAS with merely energy-distribution optimization.

wine tasting, this advantage is less pronounced. This is mainly due to the classical lower bound not being tight and uniform energy being suboptimum, as we see EAAS with energy optimization (purple) enables much better advantages, although gain optimization only leads to a slight advantage over the energy-optimized EAAS, as evident in the inset plots of Fig. 5(c),(f). In the noisy case, gain optimization enables a much better enhancement [42].

Now we address phase noise common in experiments. Phase tracking can typically eliminate the time-invariant phase noise, so the above results directly hold; when phase tracking is not possible, we can model the phase noise by adding $a_{\ell} \rightarrow e^{i \theta_{\ell}} a_{\ell}$ in Eq. (1). The random phase $\theta_{\ell}$ clearly complicates the problem. However, if we choose uniform $G=1$ (i.e., not applying OPA before photodetection), the same results of the orange curves in Fig. 5(c),(f) hold, and the classical performance can only be worse than the current benchmarks (black). Thus, the quantum advantage sustains.

Conclusion.-We have devised a near-term feasible EAAS scheme that outperforms any classical strategy in determining the presence and position of spectral absorption peaks. The EAAS scheme saturates the QCB in binary detection of a single absorption line and offers an orders-of-magnitude advantage in error probability in the discrimination of sampled spectra of molecules even in the presence of experimental nonidealities.

This research is supported by Defense Advanced Research Projects Agency (DARPA) under Young Faculty Award (YFA) Grant No. N660012014029 and University of Arizona. S. P. acknowledges funding from EU Horizon 2020 Research and Innovation Action under Grant Agreement No. 862644 (Quantum Readout Techniques and Technologies, QUARTET). Z.Z. is supported by the Office of Naval Research Grant No. N0001419-1-2190.

* Corresponding author. zhuangquntao@email.arizona.edu

[1] S. Pirandola, B. R. Bardhan, T. Gehring, C. Weedbrook, and S. Lloyd, Advances in photonic quantum sensing, Nat. Photonics 12, 724 (2018).

[2] I. R. Berchera and I. P. Degiovanni, Quantum imaging with sub-poissonian light: Challenges and perspectives in optical metrology, Metrologia 56, 024001 (2019).

[3] C. L. Degen, F. Reinhard, and P. Cappellaro, Quantum sensing, Rev. Mod. Phys. 89, 035002 (2017).

[4] V. Giovannetti, S. Lloyd, and L. Maccone, Advances in quantum metrology, Nat. Photonics 5, 222 (2011).

[5] V. Giovannetti, S. Lloyd, and L. Maccone, Quantumenhanced positioning and clock synchronization, Nature (London) 412, 417 (2001).

[6] S. Lloyd, Enhanced sensitivity of photodetection via quantum illumination, Science 321, 1463 (2008).

[7] S.-H. Tan, B. I. Erkmen, V. Giovannetti, S. Guha, S. Lloyd, L. Maccone, S. Pirandola, and J. H. Shapiro, Quantum Illumination with Gaussian States, Phys. Rev. Lett. 101, 253601 (2008).

[8] Q. Zhuang, Z. Zhang, and J. H. Shapiro, Optimum MixedState Discrimination for Noisy Entanglement-Enhanced Sensing, Phys. Rev. Lett. 118, 040801 (2017).

[9] S. Barzanjeh, S. Guha, C. Weedbrook, D. Vitali, J. H. Shapiro, and S. Pirandola, Microwave Quantum Illumination, Phys. Rev. Lett. 114, 080503 (2015).

[10] Z. Zhang, S. Mouradian, F. N. C. Wong, and J. H. Shapiro, Entanglement-Enhanced Sensing in a Lossy and Noisy Environment, Phys. Rev. Lett. 114, 110506 (2015).

[11] Q. Zhuang, Z. Zhang, and J. H. Shapiro, Entanglementenhanced lidars for simultaneous range and velocity measurements, Phys. Rev. A 96, 040304(R) (2017).

[12] S. Pirandola, Quantum Reading of a Classical Digital Memory, Phys. Rev. Lett. 106, 090504 (2011).

[13] G. Spedalieri, C. Lupo, S. L. Braunstein, and S. Pirandola, Thermal quantum metrology in memoryless and correlated environments, Quantum Sci. Technol. 4, 015008 (2018).

[14] G. Spedalieri, S. Pirandola, and S. L. Braunstein, Symmetric and asymmetric discrimination of bosonic loss: Toy applications to biological samples and photodegradable materials, Phys. Rev. A 98, 053836 (2018).

[15] Q. Zhuang, Z. Zhang, and J.H. Shapiro, Distributed quantum sensing using continuous-variable multipartite entanglement, Phys. Rev. A 97, 032329 (2018). 
[16] T. J. Proctor, P. A. Knott, and J. A. Dunningham, Multiparameter Estimation in Networked Quantum Sensors, Phys. Rev. Lett. 120, 080501 (2018).

[17] W. Ge, K. Jacobs, Z. Eldredge, A. V. Gorshkov, and M. Foss-Feig, Distributed Quantum Metrology with Linear Networks and Separable Inputs, Phys. Rev. Lett. 121, 043604 (2018).

[18] X. Guo, C. R. Breum, J. Borregaard, S. Izumi, M. V. Larsen, T. Gehring, M. Christandl, J. S. Neergaard-Nielsen, and U. L. Andersen, Distributed quantum sensing in a continuous-variable entangled network, Nat. Phys. 16, 281 (2020).

[19] Y. Xia, W. Li, W. Clark, D. Hart, Q. Zhuang, and Z. Zhang, Demonstration of a Reconfigurable Entangled RadioFrequency Photonic Sensor Network, Phys. Rev. Lett. 124, 150502 (2020).

[20] Q. Zhuang, J. Preskill, and L. Jiang, Distributed quantum sensing enhanced by continuous-variable error correction, New J. Phys. 22, 022001 (2020).

[21] LIGO Scientific Collaboration, Observation of Gravitational Waves from a Binary Black Hole Merger, Phys. Rev. Lett. 116, 061102 (2016).

[22] LIGO Scientific Collaboration, A gravitational wave observatory operating beyond the quantum shot-noise limit, Nat. Phys. 7, 962 (2011).

[23] LIGO Scientific Collaboration, Quantum-Enhanced Advanced Ligo Detectors in the Era of Gravitational-Wave Astronomy, Phys. Rev. Lett. 123, 231107 (2019).

[24] K. E. Dorfman, F. Schlawin, and S. Mukamel, Nonlinear optical signals and spectroscopy with quantum light, Rev. Mod. Phys. 88, 045008 (2016).

[25] S. Mukamel, M. Freyberger, W. P. Schleich, M. Bellini, A. Zavatta, G. Leuchs, C. Silberhorn, R. W. Boyd, L. S. Soto, A. Stefanov et al., Roadmap on quantum light spectroscopy, J. Phys. B 53, 072002 (2020).

[26] W. Demtröder, Laser Spectroscopy: Basic Concepts and Instrumentation (Springer Science \& Business Media, New York, 2013).

[27] M. Levenson, Introduction to Nonlinear Laser Spectroscopy $2 e$ (Elsevier, New York, 2012).

[28] W. E. Moerner, Nobel lecture: Single-molecule spectroscopy, imaging, and photocontrol: Foundations for superresolution microscopy, Rev. Mod. Phys. 87, 1183 (2015).

[29] O. Fischer, M. Kugler, I. Maggio-Aprile, C. Berthod, and C. Renner, Scanning tunneling spectroscopy of hightemperature superconductors, Rev. Mod. Phys. 79, 353 (2007).

[30] T. W. Hänsch and H. Walther, Laser spectroscopy and quantum opt., Rev. Mod. Phys. 71, S242 (1999).

[31] B. C. Sanders, Quantum dynamics of the nonlinear rotator and the effects of continual spin measurement, Phys. Rev. A 40, 2417 (1989).

[32] H. Lee, P. Kok, and J. P. Dowling, A quantum rosetta stone for interferometry, J. Mod. Opt. 49, 2325 (2002).

[33] H. T. Dinani, M. K. Gupta, J. P. Dowling, and D. W. Berry, Quantum-enhanced spectroscopy with entangled multiphoton states, Phys. Rev. A 93, 063804 (2016).

[34] R. Whittaker, C. Erven, A. Neville, M. Berry, J. O'Brien, H. Cable, and J. Matthews, Absorption spectroscopy at the ultimate quantum limit from single-photon states, New J. Phys. 19, 023013 (2017).
[35] M. Li, C.-L. Zou, D. Liu, G.-P. Guo, G.-C. Guo, and X.-F. Ren, Enhanced absorption microscopy with correlated photon pairs, Phys. Rev. A 98, 012121 (2018).

[36] R. Nair, Quantum-Limited Loss Sensing: Multiparameter Estimation and Bures Distance between Loss Channels, Phys. Rev. Lett. 121, 230801 (2018).

[37] D. A. Kalashnikov, A. V. Paterova, S. P. Kulik, and L. A. Krivitsky, Infrared spectroscopy with visible light, Nat. Photonics 10, 98 (2016).

[38] A. Paterova, H. Yang, C. An, D. Kalashnikov, and L. Krivitsky, Measurement of infrared optical constants with visible photons, New J. Phys. 20, 043015 (2018).

[39] A. V. Paterova, S. M. Maniam, H. Yang, G. Grenci, and L. A. Krivitsky, Quantum imaging for the semiconductor industry, Appl. Phys. Lett. 117, 054004 (2020).

[40] Coblentz Society, Inc., Evaluated infrared reference spectra, in NIST Chemistry WebBook, edited by. P. J. Linstrom and W. G. Mallard, NIST Standard Reference Database Number 69 (National Institute of Standards and Technology, Gaithersburg, MD, 2020), 20899, https://doi.org/10.18434/T4D303.

[41] C. Weedbrook, S. Pirandola, R. García-Patrón, N. J. Cerf, T. C. Ralph, J. H. Shapiro, and S. Lloyd, Gaussian quantum information, Rev. Mod. Phys. 84, 621 (2012).

[42] See Supplemental Material at http://link.aps.org/ supplemental/10.1103/PhysRevLett.125.180502 for details of analyses and additional plots, which also includes Refs. [43-49].

[43] Q. Zhuang and S. Pirandola, Entanglement-enhanced testing of multiple quantum hypotheses, Commun. Phys. 3, 103 (2020).

[44] A. Montanaro, A lower bound on the probability of error in quantum state discrimination, in 2008 IEEE Information Theory Workshop (IEEE, New York, 2008), pp. 378-380.

[45] S. J. Dolinar, Processing and transmission of information, Technical Report, Massachusetts Institute of Technology (MIT), 1973.

[46] S. Dolinar, Jr., A near-optimum receiver structure for the detection of m-ary optical ppm signals, The Telecommunications and Data Acquisition Progress Report No. 42, 1982.

[47] C. Helstrom, Quantum Detection and Estimation Theory (Academic Press, New York, 1976).

[48] J. C. Spall, An overview of the simultaneous perturbation method for efficient optimization, Johns Hopkins APL Tech. Dig. 19, 482 (1998).

[49] P. Sadegh, Constrained optimization via stochastic approximation with a simultaneous perturbation gradient approximation, Automatica 33, 889 (1997).

[50] S. Pirandola and S. Lloyd, Computable bounds for the discrimination of gaussian states, Phys. Rev. A 78, 012331 (2008).

[51] S. Pirandola, C. Lupo, V. Giovannetti, S. Mancini, and S. L. Braunstein, Quantum reading capacity, New J. Phys. 13, 113012 (2011).

[52] S. Guha and B. I. Erkmen, Gaussian-state quantumillumination receivers for target detection, Phys. Rev. A 80, 052310 (2009).

[53] H. Yu, H. Lin, H. Xu, Y. Ying, B. Li, and X. Pan, Prediction of enological parameters and discrimination of rice wine age using least-squares support vector machines and near infrared spectroscopy, J. Agric. Food Chem. 56, 307 (2008). 